\title{
Degeneration of Abelian integrals on an open Riemann surface to elliptic ones
}

\author{
By \\ Masakazu SHIBA
}

(Received Sept. 1, 1979)

An Abelian integral

$$
\Phi(x, y)=\int F(x, y) d x
$$

on a closed Riemann surface defined by an irreducible polynomial $P(x, y)=0$ is said to be reducible to an elliptic integral

$$
E(\xi, \eta)=\int G(\xi, \eta) d \xi
$$

if we can find two rational functions

$$
\xi=\xi(x, y), \quad \eta=\eta(x, y)
$$

such that

$$
\Phi(x, y)=E(\xi(x, y), \eta(x, y))
$$

or

$$
F(x, y)=G(\xi(x, y), \eta(x, y)) \frac{\frac{\partial \xi}{\partial x} \frac{\partial P}{\partial y}-\frac{\partial \xi}{\partial y} \frac{\partial P}{\partial x}}{\frac{\partial P}{\partial y}}
$$

(see Appell-Goursat [2], Baker [3] and Krazer [6]). The study of reducible Abelian integrals has as long history as the theory of hyperelliptic integrals; its origin goes back to the age of Jacobi and Legendre (cf. [3], [6], [13] etc.). But a general fundamental result was first announced by Weierstrass (see his letter to Königsberger [12]; cf. also Kowalevski [5]). He gave a necessary and sufficient condition for an Abelian integral to be reducible (to an elliptic one) in terms of its periodicity module.

Besides the above theorem Weierstrass also obtained some interesting results. In the present paper, however, we shall be concerned simply with the previous theorem. We first note that it can be easily generalized for open surfaces (see 
Theorem 1). But such an immediate generalization seems to yield no remarkable consequence. Therefore we shall restrict the case and give theorems which correspond to the classical case (see Theorems $1^{\prime}$ and 5) - it is indeed such a restricted kind of theorems that we shall need in a subsequent paper, where we shall prove another theorem of Weierstrass (and Poincaré) and give, for open surfaces of genus one, an extension of Koebe's generalized uniformization theorem for planar surfaces. In Theorems 2 and 3 we shall give some criteria for an Abelian integral to be reducible. The precise definition of "reducible" Abelian integrals, together with some other tools, will be given in the preliminary part of the paper.

Concerning the classical definition of reducibility, a few remarks should be included here. First we note that the problem of finding a reducible integral on a given surface is obviously equivalent to the problem of deciding whether there exists an analytic mapping of the surface onto a torus. Secondly, we note that Severi [9] adopts another definition; he studied not Abelian integrals themselves but their periods. Inasmuch we shall consider only the reduction to elliptic integrals, it does not matter which definition we take.

Descriminative suggestions given by $O$. Watanabe at Aichi University of Education are of use through the preparation of this paper. The author is very grateful to him.

\section{Preliminaries}

1. Throughout this paper $R$ denotes an open Riemann surface of genus $g(\leq \infty)$. Let $\partial R$ denote the Kerékjártó-Stoïlow ideal boundary of $R .^{1)}$ Set $J=\{1,2, \ldots, g\}$. By a canonical homology basis of $R \bmod \partial R$ we understand a collection of 1 -cycles $A_{j}, B_{j}(j \in J)$ on $R$ which have the following properties:

(i ) $A_{j} \times B_{k}=\delta_{j k}^{21,3)}, A_{j} \times A_{k}=B_{j} \times B_{k}=0, j, k \in J$,

(ii) $A_{j} \cap A_{k}=B_{j} \cap B_{k}=\emptyset ; A_{j} \cap B_{k}=\emptyset, j \neq k$, while $A_{j} \cap B_{j}$ consists of a single point,

(iii) there is a canonical exhaustion $\left\{R_{n}\right\}_{n=1}^{\infty}$ of $R$ such that if $0 \leq m<n$ then $\left\{A_{j}, B_{j}\right\}_{j \in J_{n} \backslash J_{m}}$ is a homology basis of $R_{n} \backslash \bar{R}_{m}$ modulo the border, ${ }^{4)}$ where $J_{n}=\left\{1,2, \ldots, g_{n}\right\}$ and $g_{n}$ is the genus of $R_{n}$.

We add an appropriate number of dividing cycles $D_{i}(i \in I)$ to $\left\{A_{j}, B_{j}\right\}_{j \in J}$ to obtain a canonical homology basis of $R$. Then every (finite) 1-cycle $\gamma$ on $R$ can be uniquely represented as

$$
\gamma \approx \sum_{j \in J}\left(m_{j} A_{j}+n_{j} B_{j}\right)+\sum_{i \in I} p_{i} D_{i} \quad(\text { a finite sum })
$$

1) Part of the results below can be easily modified to be valid for closed surfaces as well, if we interpret then $\partial R=\emptyset$.

2) The definition of the intersection number in the present paper follows the classical one and hence is different from those in Ahlfors-Sario [1] and Shiba [11] etc. .

3) $\delta_{j k}=1$ for $j=k$ and $=0$ otherwise.

4) For simplicity, we set $R_{0}=\varnothing$ and $J_{0}=\varnothing$. 
with some integers $m_{j}, n_{j}$ and $p_{i}$, where the symbol $\approx$ reads "is homologous to". We can take $D_{i}$ to be a contour of some $R_{n}$ and hence $D_{i} \cap\left(A_{j} \cup B_{j}\right)=\emptyset$ for every $(i, j) \in I \times J$. We may also assume that each of $A_{j}$ and $B_{j}$ is an analytic Jordan curve.

2. Let $\Lambda=\Lambda(R)$ be the real Hilbert space of square integrable complex differentials on $R$ (see [10] and [11]) with the inner product

$$
\left\langle\lambda_{1}, \lambda_{2}\right\rangle=\operatorname{Re} \iint_{R} \lambda_{1} \wedge \bar{\lambda}_{2}^{*}
$$

(We denote by $\bar{\lambda}$ the complex conjugate of $\lambda$ and by $\lambda^{*}$ the conjugate differential of $\lambda$. See [1], p. 266 and p. 271.) We denote by $\|\lambda\|$ the norm of $\lambda \in \Lambda:\|\lambda\|=$ $\sqrt{\langle\lambda, \lambda\rangle}$. Let $\Lambda_{h}=\Lambda_{h}(R)$ be the closed subspace of $\Lambda$ consisting of harmonic differentials on $R$. Also we set

$$
\Lambda_{e o}^{(1)}=\Lambda_{e o}^{(1)}(R)=\left\{\lambda \in \Lambda \mid \begin{array}{l|l}
{ }^{3} f \in C^{2}(R),{ }^{\exists} f_{n} \in C_{o}^{2}(R) \text { such } \\
\text { that } \lambda=d f \text { and } \lim _{n \rightarrow \infty}\left\|d f-d f_{n}\right\|=0 .
\end{array}\right\} .
$$

Hereafter we shall denote by $\boldsymbol{C}$ the set of all complex numbers (and its equivalent: Gaussian plane). For brevity, we call a straight line $L$ passing through the coordinate origin of $\boldsymbol{C}$ simply a line. Every line $L$ is determined by a $\zeta \in C^{*}=$ $\boldsymbol{C} \backslash\{0\}$ so that we shall write as

$$
L=L(\zeta)=\{z \in \boldsymbol{C} \mid z=t \zeta, t \in \boldsymbol{R}\}
$$

where $\boldsymbol{R}$ is the real number system. We also use the notation $z \equiv 0 \bmod L$ to represent $z \in L$.

A closed subspace $\Lambda_{o}$ of $\Lambda_{h}$ is called a behavior space on $\left(R,\left\{A_{j}, B_{j}\right\}_{j \in J}\right)$ associated with a family $\left\{L_{j}\right\}_{j \in J}$ of lines, if

(i) every $\lambda_{o} \in \Lambda_{o}$ is semiexact, ${ }^{5)}$

(ii) $\Lambda_{h}=\Lambda_{o} \oplus i \Lambda_{o}^{*}$ (direct sum), $i^{2}=-1$, and

(iii) for each $\lambda_{o} \in \Lambda_{o} \quad \int_{A_{j}} \lambda_{o} \equiv \int_{B_{j}} \lambda_{o} \equiv 0 \bmod L_{j}, j \in J$.

Note that we consider the family $\left\{L_{j}\right\}_{j \in J}$ to be an ordered set. Thus if $\pi$ is a permutation on $J$, then $\left\{L_{j}\right\}_{j \in J} \neq\left\{L_{\pi(j)}\right\}_{j \in J}$ in general.

Given a behavior space $\Lambda_{o}$ associated with $\left\{L_{j}\right\}_{j \in J}$, we can find a sequence $\left\{\zeta_{k}\right\}_{k=1}^{k}, \zeta_{k} \in C^{*}(1 \leq \kappa \leq \infty)$ such that (i) $\arg \zeta_{i} \neq \equiv \arg \zeta_{k}(\bmod \pi), i \neq k$ and (ii) for any $j \in J$ there is a (unique) $k=k(j), 1 \leq k \leq \kappa$ with $L_{j}=L\left(\zeta_{k}\right)$. The uniquely determined number $\kappa$ will be called the index of $\Lambda_{o}$; we shall use the notation $\kappa=$ ind $\Lambda_{o}$.

We recall now that a behavior space $\tilde{\Lambda}_{o}$ is called equivalent to $\Lambda_{o}$ if they define

5) Namely, $\int_{d} \lambda_{0}=0$ for any dividing cycle $d$ on $R$. 
the same boundary behavior (see [10]). We know that $\tilde{\Lambda}_{o}$ is equivalent to $\Lambda_{o}$ if and only if any one of $\tilde{\Lambda}_{o}$ and $\Lambda_{o}$ can be obtained from the other through a transformation (see [10], Theorem 3). A transformation is, roughly speaking, an exchange of a finite number of lines for another family. We set

$$
\text { ess. ind } \Lambda_{o}=\min \left\{\text { ind } \tilde{\Lambda}_{o} \mid \tilde{\Lambda}_{o} \text { is equivalent to } \Lambda_{o}\right\} \text {, }
$$

and call it the essential index of $\Lambda_{0}$.

3. A regular analytic (=holomorphic) differential $\psi$ on $R$ is called an Abelian differential on $R$. The integral $\Psi$ of $\psi$ is called an Abelian integral on $R$. Note that $\psi$ is not supposed to be square integrable on $R$.

Let $\Lambda_{o}$ be a behavior space on $\left(R,\left\{A_{j}, B_{j}\right\}_{j \in J}\right)$ associated with $L=\left\{L_{j}\right\}_{j \in J}$. Then a $\Lambda_{o}$-Abelian differential on $R$ is, by definition, an Abelian differential $\psi$ on $R$ such that

$$
\int_{A_{j}} \psi \equiv \int_{B_{j}} \psi \equiv 0 \bmod L_{j}
$$

for all sufficiently large $j \in J$ (cf. [11]).

We shall say that a $\Lambda_{o}$-Abelian differential $\psi$ is of the first kind if it has $\Lambda_{o}$ behavior (see [10], for instance), i.e., if there are $\lambda_{o} \in \Lambda_{o}, \lambda_{e o} \in \Lambda_{e o}^{(1)}$ such that

$$
\psi=\lambda_{o}+\lambda_{e o}
$$

outside some compact set on $R$. If a $\Lambda_{o}$-Abelian differential is semiexact, it is called of the second kind; otherwise it is called of the third kind. Such a classification of $\Lambda_{o}$-Abelian differentials obviously generalizes the classical terminology. Note that a $\Lambda_{o}$-Abelian differential of the first kind is necessarily square integrable, while $\Lambda_{o^{-}}$ Abelian differentials of the second or third kind are not always square integrable.

Let $P$ be a regular partition of $\partial R$ (see [1], p. $87 \mathrm{ff}$.). For every $n=1,2, \ldots$ we set $(P) A_{L}^{n}$ to be the class of all $(P)$ semiexact $^{6}$ ) holomorphic differential $\lambda$ on $R \backslash \bar{R}_{n}$ such that $\int_{A_{j}} \lambda \equiv \int_{B_{j}} \lambda \equiv 0 \bmod L_{j}, j \in J \backslash J_{n}$. With natural identification of its elements $(P) A_{L}=\bigcup_{n=1}^{\infty}(P) A_{L}^{n}$ has a real vector space structure. Each equivalence class of $(P) A_{L}$ modulo $\Lambda_{o}$-behavior is called a $(P) \Lambda_{o}$-singularity (cf. [10]). In this paper we shall be mainly concerned with the identity partition $I$ of $\partial R$. For brevity, we shall omit the prefix $(I) \Lambda_{o}$ whenever it is easily seen from the context that we speak of $(I) \Lambda_{o}$-singularities. For example, if $\psi$ is known to be a $\Lambda_{o}$-Abelian differential, then the singularity of $\psi$ always means the $(I) \Lambda_{o}$-singularity.

4. Suppose that $\Lambda_{o}$ is a behavior space on $\left(R,\left\{A_{j}, B_{j}\right\}_{j \in J}\right)$ associated with $\left\{L\left(\zeta_{j}\right)\right\}_{j \in J}, \zeta_{j} \in C^{*}$. Then we can find $2 g \Lambda_{o}$-Abelian differentials $\phi\left(A_{j}\right), \phi\left(B_{j}\right)$ of the first kind on $R$ such that

6) This means that $\int_{d} \varphi=0$ for every (P) dividing cycle $d$ on $R$ [which is contained in $R \backslash \bar{R}_{n}$ ]. (As usual, $(\mathrm{Q})$ semiexactness is often refered to simply as semiexactness, $\mathrm{Q}$ being the canonical partition of $\partial R$. Cf. [1]; see also footnote 5).) 


$$
\begin{aligned}
& \int_{A_{k}} \phi\left(A_{j}\right) \equiv \int_{B_{k}} \phi\left(B_{j}\right) \equiv 0 \\
& \int_{B_{k}} \phi\left(A_{j}\right) \equiv-\int_{A_{k}} \phi\left(B_{j}\right) \equiv 2 \pi i \delta_{j k} / \zeta_{k}
\end{aligned}
$$

What is more, $\phi\left(A_{j}\right)$ and $\phi\left(B_{j}\right)$ are uniquely determined by the sequence $\left\{\zeta_{j}\right\}_{j \in J}$. For the details, see [10]. We refer to these differentials as the elementary $\left[\Lambda_{o^{-}}\right.$ Abelian] differentials of the first kind determined by the sequence $\left\{\zeta_{j}\right\}_{j \in J}$.

Next let $\psi$ be a $\Lambda_{o}$-Abelian differential which is not of the first kind. Then $\psi$ is called elementary if

$$
\int_{A_{j}} \psi \equiv \int_{B_{j}} \psi \equiv 0 \bmod L\left(\zeta_{j}\right), \quad j \in J
$$

We recall the following facts ([10], [11]):

$1^{\circ}$ For any $(I) \Lambda_{o}$-singularity $\sigma \neq 0$ there is a unique elementary $\Lambda_{o}$-Abelian differential $\psi_{\sigma}{ }^{7)}$ which has the singularity $\sigma$.

$2^{\circ}$ A $\Lambda_{o}$-Abelian differential $\psi$ can be uniquely decomposed into the sum of two $\Lambda_{o}$-Abelian differentials $\psi_{o}$ and $\psi_{\sigma} ; \psi_{\sigma}$ is the elementary $\Lambda_{o}$-Abelian differential with the same singularity $\sigma$ as $\psi$ and $\psi_{o}$ is a $\Lambda_{o}$-Abelian differential of the first kind.

Henceforth we shall call $\psi_{o}$ (resp. $\psi_{\sigma}$ ) the regular (resp. singular) component of $\psi$.

5. Every behavior space $\Lambda_{o}$ has a (unique) dual behavior space (with respect to the real line $\boldsymbol{R}$ ) which we denote by $\Lambda_{o}^{\prime}$. For the precise definition, see [10]. In the present paper we only need the following facts: $\Lambda_{o}^{\prime}=\left\{\lambda \in \Lambda_{h} \mid \bar{\lambda} \in \Lambda_{o}\right\}$ and it is a behavior space on $\left(R,\left\{A_{j}, B_{j}\right\}_{j \in J}\right)$ associated with the family $\left\{L_{j}^{\prime}\right\}_{j \in J}$, where $L_{j}^{\prime}=\left\{z \in C \mid \bar{z} \in L_{j}\right\}$. A characteristic property of $\Lambda_{o}^{\prime}$ is that $\left\langle\lambda_{o}^{\prime}, i \lambda_{o}^{*}\right\rangle=0$ for all $\left(\lambda_{o}, \lambda_{o}^{\prime}\right) \in \Lambda_{o} \times \Lambda_{o}^{\prime}$ (cf. [10]).

Let $\Lambda_{o}$ be a behavior space and $\Lambda_{o}^{\prime}$ the dual behavior space (with respect to $\boldsymbol{R})$. Then for any $\Lambda_{o}^{\prime}$-Abelian integral $\Phi^{\prime}$ of the first kind and any $\Lambda_{o}$-Abelian differential $\psi$ the limit

$$
\lim _{n \rightarrow \infty} \operatorname{Re}\left[-\frac{1}{2 \pi i} \int_{\partial R_{n}} \Phi^{\prime} \psi\right]
$$

exists and is finite, which we denote by

$$
\underset{\partial R}{\operatorname{Res}} \Phi^{\prime} \psi
$$

For each $(I) \Lambda_{o}$-singularity $\sigma$

$$
\underset{\partial R}{\operatorname{Res}} \Phi^{\prime} \sigma
$$

is similarly defined by means of a representative of $\sigma$. Indeed, we can easily verify that the value does not depend on the particular choice of a representative differential

7) The $\psi_{0}$ is of the second kind if and only if $\sigma$ is semiexact. 
of $\sigma$. The quantity $\underset{\partial R}{\operatorname{Res}} \Phi^{\prime} \psi$ (resp. $\underset{\partial R}{\operatorname{Res}} \Phi^{\prime} \sigma$ ) is called the residue of $\Phi^{\prime} \psi$ (resp. $\Phi^{\prime} \sigma$ ) at the ideal boundary $\partial R$. For the details, see [11], for example.

The following proposition is a generalization of Riemann's bilinear relation and is proved by a similar method to the classical case (cf. e.g., Kusunoki [7]).

Proposition 1. Let $\Phi^{\prime}, \psi$ be as above and $\sigma$ be the singularity of $\psi .^{8)}$ Then

$$
\underset{\partial R}{\operatorname{Res}} \Phi^{\prime} \psi=\operatorname{Res}_{i R} \Phi^{\prime} \sigma=\frac{1}{2 \pi} \sum_{j \in J} \operatorname{Im}\left(\int_{A_{j}} d \Phi^{\prime} \int_{B_{j}} \psi-\int_{B_{j}} d \Phi^{\prime} \int_{A_{j}} \psi\right) .
$$

We also have (cf. [10], Lemma 1)

Proposition 1'. If $\Phi$ is a $\Lambda_{o}$-Abelian integral of the first kind, then

$$
\|d \Phi\|^{2}=-2 \sum_{j \in J} \operatorname{Im}\left(\int_{A_{j}} d \Phi \int_{B_{j}} d \bar{\Phi}\right) .
$$

The following corollary of Proposition 1 will be often used later.

Corollary. Let $\Lambda_{o}$ be a behavior space on $\left(R,\left\{A_{j}, B_{j}\right\}_{j \in J}\right)$ associated with a family $\left\{L\left(\zeta_{j}\right)\right\}_{j \in J}, \zeta_{j} \in C^{*}$, and $\Lambda_{o}^{\prime}$ be the dual behavior space (with respect to $\boldsymbol{R}$ ). Let $\sigma$ be an $(I) \Lambda_{o}$-singularity and $\psi_{\sigma}$ be the elementary $\Lambda_{o}$-Abelian differential with the singularity $\sigma$. Let $\Phi_{A_{j}}^{\prime}$ and $\Phi_{B_{j}}^{\prime}$ be the elementary $\Lambda_{o}^{\prime}$-Abelian integrals of the first kind determined by the sequence $\left\{\bar{\zeta}_{j}\right\}_{j \in J}$. Then we have

$$
\begin{aligned}
& \int_{A_{j}} \psi_{\sigma}=-\zeta_{j} \operatorname{Res}_{\partial R} \Phi_{A_{j}}^{\prime} \sigma \quad j \in J . \\
& \int_{B_{j}} \psi_{\sigma}=-\zeta_{j} \operatorname{Res}_{\partial R} \Phi_{B_{j}}^{\prime} \sigma
\end{aligned}
$$

For the proof we only need to apply Proposition 1 to $\Phi_{A_{j}}^{\prime}\left(\operatorname{resp} . \Phi_{B_{j}}^{\prime}\right)$ and $\psi_{\sigma}$.

6. Let $\Omega=\left(\omega_{1}, \omega_{2}\right) \in \boldsymbol{C}^{*} \times \boldsymbol{C}^{*}$, where $\omega_{1}, \omega_{2}$ are linearly independent over reals. We can and do always assume that the indices are so arranged that $\operatorname{Im}\left(\omega_{2} / \omega_{1}\right)$ $>0$. Denoting by $\boldsymbol{Z}$ the set of all integers, we obtain a $\boldsymbol{Z}$-module or a lattice

$$
\left[\omega_{1}, \omega_{2}\right]_{\boldsymbol{z}}=\left\{\left.z \in \boldsymbol{C}\right|^{\exists} m, n \in \boldsymbol{Z}, z=m \omega_{1}+n \omega_{2}\right\} .
$$

In order to save the notations we let the same letter $\Omega$ denote the module $\left[\omega_{1}, \omega_{2}\right]_{\mathbf{Z}}$ as well as the 2-vector $\left(\omega_{1}, \omega_{2}\right)$. Thus when we refer to a lattice it is tacitly presupposed that a basis $\left\{\omega_{1}, \omega_{2}\right\}$ is fixed so that $\operatorname{Im}\left(\omega_{2} / \omega_{1}\right)>0$.

A lineation on $R$ is, by definition, a triple $\left(\left\{A_{j}, B_{j}\right\}_{j \in J}, \varepsilon, \Omega\right)$, where $\left\{A_{j}, B_{j}\right\}_{j \in J}$ is a canonical homology basis of $R \bmod \partial R, \varepsilon$ is a mapping of $J$ into the set $\{1,2\}$ and $\Omega$ is a lattice. We shall say that a behavior space $\Lambda_{o}$ is subordinate to a lineation $\left(\left\{A_{j}, B_{j}\right\}_{j \in J}, \varepsilon, \Omega\right)$ if $\Lambda_{o}$ is a behavior space on $\left(R,\left\{A_{j}, B_{j}\right\}_{j \in J}\right)$ associated with the family $\left\{L\left(\omega_{\varepsilon(j)}\right)\right\}_{j \in J} \cdot{ }^{9)}$ Then clearly ind $\Lambda_{o} \leq 2$.

Given a lattice $\Omega=\left[\omega_{1}, \omega_{2}\right]_{\boldsymbol{z}}$, there exists a torus $T=\boldsymbol{C} / \Omega$. In such a situation

8) Here the case $\sigma=0$ is not excluded.

9) Such a behavior space was called, in [11], an $\varepsilon$-allow'able behavior space belonging to the lattice $\Omega$. 
a canonical homology basis $\left\{C_{1}, C_{2}\right\}$ of $T$ is always taken so that $C_{k}$ corresponds to the $\omega_{k}, k=1,2$, by means of the natural projection mapping.

A behavior space $\Lambda_{o}$ is said to be compatible with a torus $T$ if we can choose a lattice $\Omega$ so that (i) $T=C / \Omega$ (biholomorphically homeomorphic), and (ii) $\Lambda_{o}$ is associated with $\left\{L\left(\omega_{\varepsilon(j)}\right)\right\}_{j \in J}$ with some $\varepsilon: J \rightarrow\{1,2\}$. If a marking of $T$ is preassigned, we furthermore require that the marking coincides with that of $\boldsymbol{C} / \Omega$.

Let $\Omega=\left[\omega_{1}, \omega_{2}\right]_{z}$ be a lattice. Then a singularity $\sigma$ on $R$ is said to be $\Omega$ admissible $([11])$ if

$$
\int_{\gamma} \sigma \equiv 0 \bmod \Omega
$$

for every dividing cycle $\gamma$ which lies outside a sufficiently large compact set on $R$. Here, of course, the singularity $\sigma$ is an $(I) \Lambda_{o}$-singularity with some behavior space $\Lambda_{o}$ which need not to be compatible with the torus $C / \Omega$, and the period $\int_{\gamma} \sigma$ is computed by means of a representative differential of $\sigma$ - any choice yields the same period.

7. We shall later make use of the following proposition, which is a version of a result due to $\mathrm{K}$. Matsui.

Proposition 2. There always exists a behavior space which is subordinate to a prescribed lineation. In other words: Let a canonical homology basis $\left\{A_{j}\right.$, $\left.B_{j}\right\}_{j \in J}$ of $R \bmod \partial R$, a mapping $\varepsilon: J \rightarrow\{1,2\}$, and a 2-vector $\Omega=\left(\omega_{1}, \omega_{2}\right)$ be given, $\omega_{1}, \omega_{2} \in C^{*}, \operatorname{Im}\left(\omega_{2} / \omega_{1}\right)>0$. Then we can find a behavior space on $\left(R,\left\{A_{j}, B_{j}\right\}_{j \in J}\right)$ associated with the family $\left\{L\left(\omega_{\varepsilon(j)}\right)\right\}_{j \in J}$.

For the proof let $\Gamma_{h}=\Gamma_{h}(R)$ be the Hilbert space of square integrable real harmonic differentials on $R$ and $\Gamma_{h s e}$ its subspace which consists of semiexact differentials. Note that $\Lambda_{h}=\Gamma_{h} \oplus i \Gamma_{h}$ (direct sum) and $\Gamma_{h}=\left\{\lambda \in \Lambda_{h} \mid \lambda\right.$ is real $\}$. Set

$$
J_{k}=\{j \in J \mid \varepsilon(j)=k\}, \quad k=1,2 ;
$$

then $J_{1} \cap J_{2}=\emptyset$ and $J_{1} \cup J_{2}=J$. Let $\Gamma_{1}, \Gamma_{2}$ be two subspaces of $\Gamma_{h s e}$ which satisfy the following conditions:

$$
\begin{aligned}
& \text { (1) If } \tau \in \Gamma_{1} \text { and } j \in J_{1} \text {, then } \int_{A_{j}} \tau=\int_{B_{j}} \tau=0 . \\
& \text { (2) If } \tau \in \Gamma_{2} \text { and } j \in J_{2} \text {, then } \int_{A_{j}} \tau=\int_{B_{j}} \tau=0 \text {. } \\
& \text { (3) } \Gamma_{h}=\Gamma_{1} \oplus \Gamma_{2}^{*}=\Gamma_{1}^{*} \oplus \Gamma_{2} \text {. }
\end{aligned}
$$

As for the existence of such subspaces, consult "On the Riemann-Roch theorem on open Riemann surfaces" by the present author (J. Math. Kyoto Univ., Vol. 11 (1971)), pp. 522-524.

10) Cf. K. Matsui's forthcoming paper. The author thanks Prof. Matsui for the generosity of permitting him to include a modified proof in this paper. 
To prove the proposition ${ }^{10)}$ it now suffices to consider the case $\omega_{1}=1$ and $\omega_{2}=e^{i t}, 0<t<\pi$. We set

$$
\Lambda_{o}=e^{i t} \Gamma_{1} \oplus \Gamma_{2}
$$

and show that $\Lambda_{o}$ is a behavior space subordinate to the given lineation. Because each element $\lambda_{o}$ of $\Lambda_{o}$ is clearly semiexact and satisfies the period condition

$$
\int_{A_{j}} \lambda_{o} \equiv \int_{B_{j}} \lambda_{o} \equiv 0 \bmod L\left(\omega_{\varepsilon(j)}\right), \quad j \in J,
$$

we only have to demonstrate the orthogonal decomposition $\Lambda_{h}=\Lambda_{o} \oplus i \Lambda_{o}^{*}$.

Let $e^{i t} \theta_{1}+\theta_{2}, e^{i t} \tau_{1}+\tau_{2}$ be any two elements of $\Lambda_{o}$, where $\theta_{k}$ and $\tau_{k}$ are elements of $\Gamma_{k}, k=1,2$. Then we have

$$
\begin{aligned}
& \left\langle e^{i t} \theta_{1}+\theta_{2}, i\left(e^{i t} \tau_{1}+\tau_{2}\right)^{*}\right\rangle \\
& =\sin t\left\{\left\langle\theta_{1}, \tau_{2}^{*}\right\rangle-\left\langle\theta_{2}, \tau_{1}^{*}\right\rangle\right\}=0,
\end{aligned}
$$

so that $\Lambda_{o}$ is orthogonal to $i \Lambda_{o}^{*}$. Conversely, let $\lambda \in \Lambda_{h}$ satisfy the equation $\left\langle\lambda, i \lambda_{o}^{*}\right\rangle$ $=0$ for every $\lambda_{o}=e^{i t} \theta_{1}+\theta_{2} \in \Lambda_{o}\left(\theta_{k} \in \Gamma_{k}, k=1,2\right)$. On setting

$$
\begin{aligned}
& \theta=\frac{\lambda-\lambda}{2 i \sin t}=\frac{1}{\sin t} \operatorname{Im} \lambda \\
& \tau=\frac{e^{i t} \lambda-e^{-i t} \lambda}{2 i \sin t}=\frac{1}{\sin t} \operatorname{Im}\left(e^{i t} \lambda\right),
\end{aligned}
$$

we have

$$
\lambda=e^{i t} \theta+\tau ; \quad \theta, \tau \in \Gamma_{h} .
$$

We see that $0=\left\langle\lambda, i \lambda_{o}^{*}\right\rangle=\sin t\left\langle\left\langle\theta, \theta_{2}^{*}\right\rangle-\left\langle\tau, \theta_{1}^{*}\right\rangle\right\}$ holds for any pair $\left(\theta_{1}, \theta_{2}\right) \in$ $\Gamma_{1} \times \Gamma_{2}$. Since $\Gamma_{h}=\Gamma_{1} \oplus \Gamma_{2}^{*}=\Gamma_{1}^{*} \oplus \Gamma_{2}$, it follows that $\theta \in \Gamma_{1}$ and $\tau \in \Gamma_{2}$. Hence we have proved the proposition.

\section{Results and proofs}

8. We shall begin with the following

Definition 1. An Abelian integral $\Psi$ on $R$ is called quasi-reducible if there are a torus $T$, a holomorphic integral $E$ on $T$, and an analytic mapping $f$ of $R$ into $T$ such that

$$
\Psi=E \circ f^{11)} \text {. }
$$

Note that we retain the term "reducible" for a more restricted situation (see Definition 2 below), since we can easily prove

11) Namely, $\Psi$ is quasi-reducible if and only if $d \Psi$ can be represented as the pull-back of a holomorphic differential $d E$ on a torus $T$ via an analytic mapping $f: d \Psi=f^{*}(d E)$. 
Theorem 1. Let $\left\{A_{j}, B_{j} ; D_{i}\right\}_{j \in J, i \in I}$ be an arbitrarily fixed canonical homology basis of $R$. Then an Abelian integral $\Psi$ on $R$ is quasi-reducible if and only if we can find a lattice $\Omega=\left[\omega_{1}, \omega_{2}\right]_{z}$ and integers $m_{j k}, n_{j k}, p_{i k}(j \in J, i \in I ; k=1,2)$ such that

$$
\begin{aligned}
& \int_{A_{j}} d \Psi=m_{j 1} \omega_{1}+m_{j 2} \omega_{2} \\
& \int_{B_{j}} d \Psi=n_{j 1} \omega_{1}+n_{j 2} \omega_{2} \quad j \in J, i \in I . \\
& \int_{D_{i}} d \Psi=p_{i 1} \omega_{1}+p_{i 2} \omega_{2}
\end{aligned}
$$

If this is the case, $\Psi$ is quasi-reducible to a holomorphic integral on the torus $\mathrm{C} / \Omega$.

The proof is similar to that of the compact case (see Appell-Goursat [2] and Krazer [6]):

First of all, let $\Psi$ be a quasi-reducible Abelian integral on $R$ and $E$ be an holomorphic integral on a torus $T$, to which $\Psi$ is quasi-reduced by an analytic mapping $f: R \rightarrow T$. Taking a canonical homology basis $\left\{C_{1}, C_{2}\right\}$ of $T$, we set

$$
\omega_{k}=\int_{C_{k}} d E, \quad k=1,2 .
$$

Since we have $\int_{\gamma} d \Psi=\int_{f(\gamma)} d E$ for every cycle $\gamma$ on $R$, we can find integers $m_{j k}, n_{j k}$, $p_{i k}(j \in J, i \in I ; k=1,2)$ such that

$$
\left\{\begin{array}{l}
\int_{A_{j}} d \Psi=m_{j 1} \omega_{1}+m_{j 2} \omega_{2} \\
\int_{B_{j}} d \Psi=n_{j 1} \omega_{1}+n_{j 2} \omega_{2} \\
\int_{D_{i}} d \Psi=p_{i 1} \omega_{1}+p_{i 2} \omega_{2}
\end{array} \quad j \in J, i \in I .\right.
$$

Suppose, conversely, we are given a lattice $\Omega=\left[\omega_{1}, \omega_{2}\right]_{z}$ and an Abelian integral $\Psi$ which satisfy equations (*). Denoting by $T$ the torus $C / \Omega$ and by $\rho$ the natural projection mapping of $\boldsymbol{C}$ onto $T$ (a holomorphic universal covering mapping), we can easily verify that $f=\rho \circ \Psi$ is a well-defined analytic mapping of $R$ into $T$. The inverse $\rho^{-1}$ of $\rho$ is locally single-valued and $d \rho^{-1}$ is a holomorphic differential on $T$, which we will denote by $d E$. Then we can write as $d \Psi=f^{*}(d E)$ so that $\Psi$ is quasireducible.

If $\partial R \neq \emptyset$ actually, a famous theorem due to Behnke and Stein (see BehnkeSommer [4], Satz 10, S. 451) together with the above Theorem 1 implies that there are a plenty of quasi-reducible Abelian integrals on $R$. We also notice that the existence of a quasi-reducible Abelian integral on an open Riemann surface induces, in contrast to the classical case, no special characteristic property of the surface. 
It will still make sense to ask whether a given $\Lambda_{o}$-Abelian integral is quasireducible or not. Concerning this problem, we have the following proposition, which can be easily deduced from Theorem 1 and Corollary to Proposition 1.

Proposition 3. Let $\Lambda_{0}$ be a behavior space on $\left(R,\left\{A_{j}, B_{j}\right\}_{j \in J}\right)$ associated with a family $\left\{L\left(\zeta_{j}\right)\right\}_{j \in J}, \zeta_{j} \in C^{*}$, and $\Lambda_{o}^{\prime}$ be the dual behavior space. Let $\Phi_{A_{j}}^{\prime}$ and $\Phi_{B_{j}}^{\prime}$ be the elementary $\Lambda_{o}^{\prime}$-Abelian integrals of the first kind determined by the sequence $\left\{\bar{\zeta}_{j}\right\}_{j \in J}$. Finally, let $\Omega$ be any lattice. Then a $\Lambda_{o}$-Abelian integral $\Psi=\Psi_{o}+\Psi_{\sigma}$ is quasi-reducible to a holomorphic integral on the torus $C / \Omega$ if and only if

(i) $\sigma$ is $\Omega$-admissible, and

(ii) $\int_{A j} d \Psi_{o} \equiv \zeta_{j} \operatorname{Res}_{\partial R} \Phi_{A j}^{\prime} \sigma$

$$
\int_{B_{j}} d \Psi_{o} \equiv \zeta_{j} \operatorname{Res}_{\partial R} \Phi_{B_{j}}^{\prime} \sigma
$$

$\bmod \Omega, j \in J$

9. Our problem is now to characterize, in connection with behavior spaces, an Abelian integral which is the pull-back of a holomorphic integral on a torus via an analytic mapping of more restricted nature. The restriction which we shall impose on the mapping is described in terms of the homological property of the mapping.

To be more precise, let $R$ be as before with a fixed canonical basis $\Xi(R)=$ $\left\{A_{j}, B_{j}\right\}_{j \in J} \bmod \partial R$. Let $T$ be a torus and $\Xi(T)=\left\{C_{1}, C_{2}\right\}$ a canonical homology basis of $T$. A continuous mapping $f: R \rightarrow T$ is called of finite (resp. null) type relative to $(\Xi(R), \Xi(T))$ if

$$
\sum_{j \in J} \prod_{v=1,2}\left\{\left(f\left(A_{j}\right) \times C_{v}\right)^{2}+\left(f\left(B_{j}\right) \times C_{v}\right)^{2}\right\}
$$

is finite (resp. is equal to zero). Cf. [10].

Using these terms, we have now another

Definition 2. If, in Definition 1, the mapping $f$ can be chosen so as to be of finite (resp. null) type relative to some pair of canonical bases $(\Xi(R), \Xi(T))$, then $\Psi$ is called reducible (resp. strongly reducible). If it is necessary to refer to the basis $\Xi(R)$ used, we say that $\Psi$ is reducible (resp. strongly reducible) with respect to $\Xi(R)$.

From the definitions we have immediately

(a) A reducible Abelian integral is quasi-reducible.

(a') In case $g<\infty$, the converse of (a) is also true.

(b) A strongly reducible Abelian integral is reducible.

On the analogy of Theorem 1 we can prove the following theorem (cf. [2], [3], [5], [6] and [12]).

Theorem 1'. Let $\left\{A_{j}, B_{j} ; D_{i}\right\}_{j \in J, i \in I}$ be a canonical homology basis of $R$. Then an Abelian integral $\Psi$ on $R$ is reducible with respect to $\left\{A_{j}, B_{j}\right\}_{j \in J}$ if and only if there exist integers $m_{j k}, n_{j k}$ and $p_{i k}(j \in J, i \in I ; k=1,2)$ and two complex 
numbers $v_{1}, v_{2}$ such that

(i) $\int_{A_{j}} d \Psi=m_{j 1} v_{1}+m_{j 2} v_{2}$

$j \in J$,

$\int_{B_{j}} d \Psi=n_{j 1} v_{1}+n_{j 2} v_{2}$

(i') $\int_{D_{i}} d \Psi=p_{i 1} v_{1}+p_{i 2} v_{2} \quad i \in I$,

(ii) $\sum_{j \in J}\left(m_{j 1}^{2}+n_{j 1}^{2}\right)\left(m_{j 2}^{2}+n_{j 2}^{2}\right)<\infty$,

(iii) $v_{1}, v_{2} \neq 0, \quad \operatorname{Im}\left(v_{2} / v_{1}\right) \neq 0$.

10. The following proposition is an easy consequence of Theorem $1^{\prime}$ and Proposition 2 (cf. the proof of Proposition 6).

Proposition 4. If $\Psi$ is a [nonconstant] Abelian integral on $R$ which is reducible with respect to a basis $\left\{A_{j}, B_{j}\right\}_{j \in J} \bmod \partial R$, then there exists a behavior space $\Lambda_{o}$ on $\left(R,\left\{A_{j}, B_{j}\right\}_{j \in J}\right)$ such that $\Psi$ is $\Lambda_{o}$-Abelian.

Thus every reducible Abelian integral on $R$ is $\Lambda_{0}$-Abelian with a suitably chosen behavior space $\Lambda_{o}$ and hence we can speak of the singularity of a reducible Abelian integral. Also, it makes sense to characterize the possible singularities of reducible Abelian integrals. We shall discuss this problem later; see Theorems 2, 3 and 4 below.

We also have

Proposition 5. Let $T$ be a torus and $\Lambda_{o}$ a behavior space on $\left(R,\left\{A_{j}, B_{j}\right\}_{j \in J}\right)$ compatible with $T$. Then a $\Lambda_{o}$-Abelian integral on $R$ which is quasi-reducible to a holomorphic integral on $T$ is reducible.

Proof. Suppose that $\Psi$ is a $\Lambda_{o}$-Abelian integral and is quasi-reducible to a holomorphic integral on $T$. Since $\Lambda_{o}$ is compatible with $T$, we can find a lattice $\Omega=\left[\omega_{1}, \omega_{2}\right]_{z}$ and a mapping $\varepsilon: J \rightarrow\{1,2\}$ such that

(i) $T=C / \Omega$, and

(ii) $\Lambda_{o}$ is associated with the family $\left\{L\left(\omega_{\varepsilon(j)}\right)\right\}_{j \in J}$.

If we set $\varepsilon^{*}(j)=3-\varepsilon(j), j \in J, \varepsilon^{*}$ is also a mapping of $J$ into the set $\{1,2\}$, and $\varepsilon^{*}(j)$ $\neq \varepsilon(j)$ for all $j \in J$. By Theorem 1 we have

$$
\begin{aligned}
& \int_{A_{j}} d \Psi=m_{j \varepsilon(j)} \omega_{\varepsilon(j)}+m_{j \varepsilon^{*}(j)} \omega_{\varepsilon^{*}(j)} \\
& \int_{B_{j}} d \Psi=n_{j \varepsilon(j)} \omega_{\varepsilon(j)}+n_{j \varepsilon^{*}(j)} \omega_{\varepsilon^{*}(j)} \quad j \in J, \quad i \in I, \\
& \int_{D_{i}} d \Psi=p_{i 1} \omega_{1}+p_{i 2} \omega_{2}
\end{aligned}
$$


with some integers $m_{j k}, n_{j k}, p_{i k}(j \in J, i \in I ; k=1,2)$. Because $\Psi$ is a $\Lambda_{o}$-Abelian integral and $\operatorname{Im}\left(\omega_{2} / \omega_{1}\right)>0$, we see that

$$
m_{j \varepsilon^{*}(j)}=n_{j \varepsilon^{*}(j)}=0
$$

except for a finite number of $j$. It now follows from Theorem $1^{\prime}$ that $\Psi$ is reducible.

q.e.d.

11. In connection with Proposition 4 we shall prove the following

Proposition 6. Let $\Psi$ be a strongly reducible non-constant Abelian integral on $R$. Then there is a behavior space $\Lambda_{o}($ on $R)$ such that $\Psi$ is a $\Lambda_{o}$-Abelian integral which is not of the first kind.

Proof. Since $\Psi$ is strongly reducible, there are a torus $T$, a holomorphic integral $E$ on $T$, and an analytic mapping $f: R \rightarrow T$ which have the following properties:

(i) $d \Psi=f^{*}(d E)$,

(ii) there are canonical homology bases $\left\{A_{j}, B_{j}\right\}_{j \in J}(\bmod \partial R)$ and $\left\{C_{1}, C_{2}\right\}$ on

$R$ and $T$, respectively, such that $f$ is of null type relative to $\left(\left\{A_{j}, B_{j}\right\}_{j \in J},\left\{C_{1}, C_{2}\right\}\right)$. Set $\omega_{k}=\int_{C_{k}} d E, k=1,2$, and $\Omega=\left[\omega_{1}, \omega_{2}\right]_{z}$. We have then (cf. the proof of Theorem 1)

$$
\begin{aligned}
& \int_{A_{j}} d \Psi=m_{j 1} \omega_{1}+m_{j 2} \omega_{2} \\
& \int_{B_{j}} d \Psi=n_{j 1} \omega_{1}+n_{j 2} \omega_{2}
\end{aligned} \quad j \in J,
$$

where $m_{j k}, n_{j k}$ are appropriate integers satisfying

$$
\sum_{j \in J}\left(m_{j 1}^{2}+n_{j 1}^{2}\right)\left(m_{j 2}^{2}+n_{j 2}^{2}\right)=0 .
$$

If we set

$$
\varepsilon(j)= \begin{cases}1 & \text { if } m_{j 2}=n_{j 2}=0 \\ 2 & \text { otherwise }\end{cases}
$$

then $\varepsilon$ is a well-defined mapping of $J$ into the set $\{1,2\}$. Proposition 2 ensures that there is a behavior space $\Lambda_{o}$ which is subordinate to the lineation $\left(\left\{A_{j}, B_{j}\right\}_{j \in J}, \varepsilon, \Omega\right)$. The integral $\Psi$ is obviously $\Lambda_{o}$-Abelian. (The discussion so far constitutes, with a few necessary modifications, a proof of Proposition 4.)

If $\Psi$ were of the first kind, $\Psi$ would be constant; for then by Proposition 1' we would have

$$
\begin{aligned}
\|d \Psi\|^{2} & =-2 \sum_{j \in J} \operatorname{Im}\left(\int_{A_{j}} d \Psi \int_{B_{j}} d \bar{\Psi}\right) \\
& =-2 \operatorname{Im}\left(\omega_{1} \bar{\omega}_{2}\right) \sum_{j \in J}\left(m_{j 1} n_{j 2}-m_{j 2} n_{j 1}\right)=0
\end{aligned}
$$

a contradiction. q.e.d. 
We can also prove

Proposition 7. Let $\Lambda_{o}$ be a behavior space on $\left(R,\left\{A_{j}, B_{j}\right\}_{j \in J}\right)$. Then there is no non-constant $\Lambda_{o}$-Abelian integral of the first kind which is strongly reducible with respect to the basis $\left\{A_{j}, B_{j}\right\}_{j \in J}$.

The proof is omitted (cf. [11]). It should be noted that $\Lambda_{o}$ may be quite arbitrary; in particular, the (essential) index of $\Lambda_{o}$ is not necessarily less than or equal to two.

12. In the remaining of the paper we shall give some conditions for an Abelian integral on $R$ to be reducible. We shall first prove a refinement of Proposition 4.

Theorem 2. Suppose that $\Psi$ is a reducible Abelian integral on $R$. Then we can find a lineation $\left(\left\{A_{j}, B_{j}\right\}_{j \in J}, \varepsilon,\left(\omega_{1}, \omega_{2}\right)\right)$ which has the following properties: For any behavior space $\Lambda_{o}$ which is subordinate to the lineation

(A) $\Psi$ is a $\Lambda_{o}$-Abelian integral.

(B) The singularity $\sigma$ of $d \Psi$ is $\left[\omega_{1}, \omega_{2}\right]_{z}$-admissible.

(C) The regular component $d \Psi_{\circ}$ of $d \Psi$ satisfies

$$
\begin{aligned}
& \int_{A_{j}} d \Psi_{o} \equiv \omega_{\varepsilon(j)} \operatorname{Res}_{\partial R} \Phi_{A_{j}}^{\prime} \sigma \\
& \int_{B_{j}} d \Psi_{o} \equiv \omega_{\varepsilon(j)} \operatorname{Res}_{\partial R} \Phi_{B_{j}}^{\prime} \sigma
\end{aligned}
$$

Here, $\Phi_{A_{j}}^{\prime}$ and $\Phi_{B_{j}}^{\prime}$ are the elementary $\Lambda_{o}^{\prime}$-Abelian integrals of the first kind determined by the sequence $\left\{\bar{\omega}_{\varepsilon(j)}\right\}_{j \in J}, \Lambda_{o}^{\prime}$ being the dual behavior space of $\Lambda_{o}$.

Proof. Since $\Psi$ is reducible, there are a torus $T$, a holomorphic integral $E$ on $T$, and an analytic mapping $f: R \rightarrow T$ of finite type such that $\Psi=E \circ f$. Let $\left(\left\{A_{j}\right.\right.$, $\left.\left.B_{j}\right\}_{j \in J},\left\{C_{1}, C_{2}\right\}\right)$ be a pair of canonical homology bases of $R(\bmod \partial R)$ and $T$ relative to which $f$ is of finite type. Namely, there is a finite subset $J_{o}$ of $J$ such that

$$
\begin{aligned}
& f\left(A_{j}\right) \approx m_{j} C_{v_{j}} \\
& f\left(B_{j}\right) \approx n_{j} C_{v_{j}}
\end{aligned}
$$

where $m_{j}, n_{j}$ are integers and $v_{j}$ is 1 or 2 depending on $j$. Setting

$$
\varepsilon(j)= \begin{cases}v_{j} & \text { for } j \in J \backslash J_{o} \\ 1 & \text { for } j \in J_{o}\end{cases}
$$

and

$$
\omega_{k}=\int_{C_{k}} d E, \quad k=1,2,
$$

we obtain a mapping $\varepsilon$ of $J$ into $\{1,2\}$ and a lattice $\Omega=\left[\omega_{1}, \omega_{2}\right]_{z}$. We claim that $\left(\left\{A_{j}, B_{j}\right\}_{j \in J}, \varepsilon, \Omega\right)$ is a lineation for which we are looking.

Take an arbitrary behavior space $\Lambda_{o}$ which is subordinate to $\left(\left\{A_{j}, B_{j}\right\}_{j \in J}, \varepsilon, \Omega\right)$. 
Such a behavior space $\Lambda_{o}$ surely exists (Proposition 2). Then $\Psi$ is a $\Lambda_{o}$-Abelian integral, for $\Lambda_{o}$ is associated with the family $\left\{L\left(\omega_{\varepsilon(j)}\right)\right\}_{j \in J}$ and the differential $d \Psi$ satisfies

$$
\begin{aligned}
& \int_{A_{j}} d \Psi=\int_{f\left(A_{j}\right)} d E=m_{j} \omega_{\varepsilon(j)} \equiv 0 \\
& \int_{B_{j}} d \Psi=\int_{f_{\left(B_{j}\right)} d E=n_{j} \omega_{\varepsilon(j)} \equiv 0}
\end{aligned}
$$

Since $\Psi$ is reducible and hence quasi-reducible to $E$ which is a holomorphic integral on the torus $T=\boldsymbol{C} / \Omega$, we have by Proposition 3

$$
\begin{aligned}
& \int_{A_{j}} d \Psi_{o} \equiv \omega_{\varepsilon(j)} \operatorname{Res}_{\partial R} \Phi_{A_{j}}^{\prime} \sigma \\
& \int_{B_{j}} d \Psi_{o} \equiv \omega_{\varepsilon(j)} \operatorname{Res}_{\partial R} \Phi_{B_{j}}^{\prime} \sigma
\end{aligned}
$$

(Compare the proof of Theorem 1 in Shiba [11].) The remaining assertion (B) is obvious by Theorem 1'.

q.e.d.

13. The following theorem gives a sort of converse of Theorem 2 .

Theorem 3. Let $\Psi$ be an Abelian integral on $R$. Suppose there exist a lineation and a behavior space $\Lambda_{o}$ subordinate to the lineation such that (A), (B) and (C) are fulfilled. Then $\Psi$ is reducible.

We shall only give a sketch of the proof. In the first place, Proposition 3 implies that $\Psi$ is quasi-reducible to a holomorphic integral on the torus $C / \Omega$. Since $\Lambda_{o}$ is clearly compatible with $\boldsymbol{C} / \Omega$ and $\Psi$ is $\Lambda_{o}$-Abelian (condition (A)), we see by Proposition 5 that $\Psi$ is reducible.

For convenience' sake we reformulate Theorems 2 and 3 in the following

Corollary. An Abelian integral $\Psi$ on $R$ is reducible if and only if there exist a lineation $\left(\left\{A_{j}, B_{j}\right\}_{j \in J}, \varepsilon, \Omega\right)$ and a behavior space $\Lambda_{o}$ subordinate to the lineation such that (A), (B) and (C) are satisfied.

Note that the use of Proposition 5 in the proof of above Theorem 3 is dispensable. We then observe that Theorem 3 together with Proposition 3 gives another proof of Proposition 5. To see this, let $T$ and $\Lambda_{o}$ be the same as in Proposition 5. Let $\left(\left\{A_{j}, B_{j}\right\}_{j \in J}, \varepsilon, \Omega\right)$ be a lineation, $\Omega=\left(\omega_{1}, \omega_{2}\right)$, such that

(i) $T=C / \Omega$, and

(ii) $\Lambda_{o}$ is subordinate to the lineation.

Let $\Psi$ be a $\Lambda_{o}$-Abelian integral which is quasi-reducible to a holomorphic integral on $T$ and $\sigma$ be the singularity of $d \Psi$. Decompose $d \Psi$ into its regular and singular components: $d \Psi=d \Psi_{o}+d \Psi_{\sigma}$. Then Proposition 3 implies that $\sigma$ is $\Omega$-admissible and that 


$$
\begin{aligned}
& \int_{A_{j}} d \Psi_{o} \equiv \omega_{\varepsilon(j)} \operatorname{Res}_{\partial R} \Phi_{A_{j}}^{\prime} \sigma \\
& \int_{B_{j}} d \Psi_{o} \equiv \omega_{\varepsilon(j)} \operatorname{Res}_{\partial R} \Phi_{B_{j}}^{\prime} \sigma
\end{aligned}
$$

$\Phi_{A_{j}}^{\prime}$ and $\Phi_{B_{j}}^{\prime}$ being the elementary $\Lambda_{o}^{\prime}$-Abelian integrals of the first kind determined by the sequence $\left\{\bar{\omega}_{\varepsilon(j)}\right\}_{j \in J}$. Thus conditions (B) and (C) are also fulfilled. We can now use Theorem 3 to conclude that $\Psi$ is reducible. So we have proved Proposition 5 .

14. We will call an $(I) \Lambda_{o}$-singularity $\sigma$ reducible if there is a reducible $\Lambda_{o^{-}}$ Abelian integral with the singularity $\sigma$. Then we can prove

Theorem 4. Let $\Lambda_{o}$ be a behavior space subordinate to the lineation $\left(\left\{A_{j}, B_{j}\right\}_{j \in J}\right.$, $\varepsilon, \Omega), \Omega=\left(\omega_{1}, \omega_{2}\right)$. Then an $(I) \Lambda_{o}$-singularity $\sigma$ is reducible if and only if

(D) $\sigma$ is $\Omega$-admissible;

(E) There exists a $\Lambda_{o}$-Abelian differential $\phi$ of the first kind such that

$$
\begin{aligned}
& \int_{A_{j}} \phi \equiv \omega_{\varepsilon(j)} \operatorname{Res}_{\partial R} \Phi_{A_{j}}^{\prime} \sigma \\
& \int_{B_{j}} \phi \equiv \omega_{\varepsilon(j)} \operatorname{Res}_{\partial R} \Phi_{B_{j}}^{\prime} \sigma
\end{aligned}
$$

Proof. Assume, first of all, (D) and (E). (We could make use of the reasoning used in the proof of Theorem 3, but here we apply Abel's theorem (see [11]) in order to point out the explicit interrelation of these two topics.) Denote, as usual, by $\psi_{\sigma}$ the (unique) elementary $\Lambda_{0}$-Abelian differential with the singularity $\sigma$ (cf. section 4 , $1^{\circ}$ ). Let $T$ be the torus $C / \Omega$ and $\left\{C_{1}, C_{2}\right\}$ be its canonical homology basis which is induced by the lattice $\Omega$ (see section 6). Let $E_{o}$ be the normalized holomorphic integral on $T$ with respect to $\left\{C_{1}, C_{2}\right\}$, i.e., suppose $\int_{C_{1}} d E_{o}=1$. Then $\int_{C_{2}} d E_{0}=$ $\omega_{2} / \omega_{1}$. Setting $E=\omega_{1} E_{o}$, we know by Theorem 1 in [11] that conditions (D) and (E) imply the existence of an analytic mapping $f: R \rightarrow T$ such that

(i) $f$ is of finite type relative to $\left(\left\{A_{j}, B_{j}\right\}_{j \in J},\left\{C_{1}, C_{2}\right\}\right)$, and

(ii) $f^{*}(d E)-\psi_{\sigma}$ is of the first kind.

Therefore the integral of $f^{*}(d E)=\left(f^{*}(d E)-\psi_{\sigma}\right)+\psi_{\sigma}$ is a reducible $\Lambda_{o}$-Abelian integral with the singularity $\sigma$. Hence $\sigma$ is reducible. The converse follows easily from the same Theorem 1 in [11], so that we have completed the proof.

Let $\Lambda_{o}$ be an arbitrary behavior space on $R$. Then an $(I) \Lambda_{o}$-singularity $\sigma$ will be called quasi-reducible if there is a quasi-reducible $\Lambda_{o}$-Abelian integral on $R$ which has the singularity $\sigma$. The following proposition is an immediate consequence of Proposition 3. Compare the preceding theorem, too.

Proposition 8. Let $\Lambda_{o}$ be a behavior space on $\left(R,\left\{A_{j}, B_{j}\right\}_{j \in J}\right)$ associated with the family $\left\{L\left(\zeta_{j}\right)\right\}_{j \in J}$. Then an $(I) \Lambda_{o}$-singularity $\sigma$ is quasi-reducible if and only 
if there are a lattice $\Omega$ and $a \Lambda_{o}$-Abelian differential $\phi$ of the first kind such that $\sigma$ is $\Omega$-admissible and

$$
\begin{aligned}
& \int_{A_{j}} \phi \equiv \zeta_{j} \operatorname{Res}_{\partial R} \Phi_{A_{j}}^{\prime} \sigma \\
& \int_{B_{j}} \phi \equiv \zeta_{j} \operatorname{Res}_{\partial R} \Phi_{B_{j}}^{\prime} \sigma
\end{aligned}
$$

Here, of course, $\Phi_{A_{j}}^{\prime}$ and $\Phi_{B_{j}}^{\prime}$ stand for the elementary $\Lambda_{o}^{\prime}$-integrals of the first kind determined by the sequence $\left\{\zeta_{j}\right\}_{j \in J}$.

15. In this section we shall be concerned with Abelian integrals of the first kind. Notice that the term "of the first kind" is meaningless unless we fix a behavior space on $R$.

First we shall prove the following generalization of Weierstrass's theorem (see [5], [12]; cf. also [2], [3] and [6]).

Theorem 5. Let $\Lambda_{o}$ be a behavior space on $\left(R,\left\{A_{j}, B_{j}\right\}_{j \in J}\right)$ and $\Phi$ be a nonconstant $\Lambda_{o}$-Abelian integral of the first kind. Then $\Phi$ is reducible with respect to $\left\{A_{j}, B_{j}\right\}_{j \in J}$ if and only if there are $v_{k} \in C ; m_{j k}, n_{j k} \in Z(k=1,2 ; j \in J)$ such that

(i) $\int_{A_{j}} d \Phi=m_{j 1} v_{1}+m_{j 2} v_{2}$

$$
\int_{B_{j}} d \Phi=n_{j 1} v_{1}+n_{j 2} v_{2} \quad j \in J,
$$

(ii) $\sum_{j \in J}\left(m_{j 1}^{2}+n_{j 1}^{2}\right)\left(m_{j 2}^{2}+n_{j 2}^{2}\right)<\infty$.

Proof. The only if part is proved in Theorem 1', where we have actually shown that the numbers $v_{1}, v_{2}$ are subject to a more restricted condition (see (iii) in Theorem $1^{\prime}$ ). To prove the if part it suffices to verify that $v_{1}, v_{2}$ satisfy condition (iii) in Theorem 1'. But this is a simple consequence of Proposition 1'. In fact, we have

$$
\begin{aligned}
0 & <\|d \Phi\|^{2}=-2 \sum_{j \in J} \operatorname{Im}\left(\int_{A_{j}} d \Phi \int_{B_{j}} d \bar{\Phi}\right) \\
& =-2 \operatorname{Im}\left(v_{1} \bar{v}_{2}\right) \sum_{j \in J}\left(m_{j 1} n_{j 2}-m_{j 2} n_{j 1}\right),
\end{aligned}
$$

so that neither $v_{1}$ nor $v_{2}$ vanishes and $\operatorname{Im}\left(v_{1} \bar{v}_{2}\right) \neq 0$.

q.e.d.

Now we recall that two behavior spaces on $R$ are said to be equivalent if they define the same boundary behavior (see [10]). We have then

Theorem 6. Let $\Lambda_{o}$ be a behavior space on $\left(R,\left\{A_{j}, B_{j}\right\}_{j \in J}\right)$. Suppose that, among the $\Lambda_{o}$-Abelian integrals of the first kind, there is a reducible $\Phi$ with respect

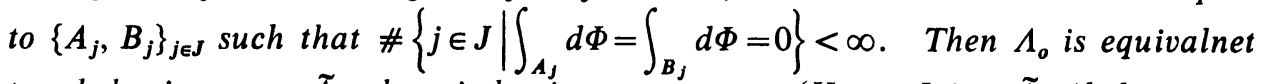
to a behavior space $\tilde{\Lambda}_{o}$ whose index is at most two. (Hence $\Phi$ is a $\tilde{\Lambda}_{o}$-Abelian inte- 
gral of the first kind. Also, ess. ind $\Lambda_{o} \leq 2$.)

Proof. Evidently, it suffices to consider the case $g=\infty$; so we assume that $R$ is of infinite genus. Let $\left\{L_{j}\right\}_{j \in J}$ be the family of lines with which $\Lambda_{o}$ is associated. Then we can find a positive integer $j_{1}$ such that

$$
\int_{A_{j}} d \Phi \equiv \int_{B_{j}} d \Phi \equiv 0 \quad \bmod L_{j}, \quad j>j_{1},
$$

for $\Phi$ is a $\Lambda_{o}$-Abelian integral. On the other hand, since $\Phi$ is reducible with respect to $\left\{A_{j}, B_{j}\right\}_{j \in J}$, we can find $m_{j k}, n_{j k} \in Z$ and $v_{1}, v_{2} \in C^{*}, \operatorname{Im}\left(v_{2} / v_{1}\right)>0$ (cf. Theorems $1^{\prime}$ and 5) such that

$$
\begin{aligned}
& \int_{A_{j}} d \Phi=m_{j 1} v_{1}+m_{j 2} v_{2} \\
& \int_{B_{j}} d \Phi=n_{j 1} v_{1}+n_{j 2} v_{2}
\end{aligned} \quad j \in J
$$

and

$$
\sum_{j \in J}\left(m_{j 1}^{2}+n_{j 1}^{2}\right)\left(m_{j 2}^{2}+n_{j 2}^{2}\right)<\infty .
$$

Hence there exists a positive integer $j_{2}$ such that if $j>j_{2}$ then either $m_{j 1}=n_{j 1}=0$ or $m_{j 2}=n_{j 2}=0$.

For $j \in J$ we set $\varepsilon(j)=1$ if $m_{j 2}=n_{j 2}=0$ and $\varepsilon(j)=2$ otherwise. If we set furthermore $L_{k}^{*}=L\left(v_{k}\right), k=1,2$, we have

$$
\int_{A_{j}} d \Phi \equiv \int_{B_{j}} d \Phi \equiv 0 \quad \bmod L_{\varepsilon(j)}^{*}, \quad j>j_{2} .
$$

By assumption, if $j$ is sufficiently large, say, $j>j_{3}$, then $\left|\int_{A_{j}} d \Phi\right|+\left|\int_{B_{j}} d \Phi\right| \neq 0$; and hence we see

$$
L_{j}=L_{\varepsilon(j)}^{*} \quad \text { for } j>j_{o},
$$

$j_{o}$ being the maximum of $j_{1}, j_{2}$ and $j_{3}$.

Let $J^{*}=\left\{1,2, \ldots, j_{0}\right\}$ and set

$$
\tilde{L}_{j}= \begin{cases}L_{j} & \text { if } j \in J \backslash J^{*} \\ L_{\varepsilon(j)}^{*} & \text { if } j \in J^{*} .\end{cases}
$$

In [10] we proved that $\tilde{\Lambda}_{o}=\left\{\lambda \in \Lambda_{h} \mid \lambda\right.$ has $\Lambda_{o}$-behavior and $\int_{A_{j}} \lambda \equiv \int_{B_{j}} \lambda \equiv 0 \bmod \tilde{L}_{j}$, $j \in J\}$ is also a behavior space on $\left(R,\left\{A_{j}, B_{j}\right\}_{j \in J}\right)$ and that $\tilde{\Lambda}_{o}$ is equivalent to the $\Lambda_{o}$. The $\tilde{\Lambda}_{o}$ thus obtained is subordinate to the lineation $\left(\left\{A_{j}, B_{j}\right\}_{j \in J}, \varepsilon,\left(v_{1}, v_{2}\right)\right)$ and so ind $\tilde{\Lambda}_{o} \leq 2$. Finally, by Proposition 8 in [10], the integral $\Phi$ may well be regarded as a $\tilde{\Lambda}_{o}$-Abelian integral of the first kind.

q.e.d.

Corollary. Let $R$ be an open Riemann surface of infinite genus. If $\Lambda_{o}$ is a behavior space on $\left(R,\left\{A_{j}, B_{j}\right\}_{j \in J}\right)$ such that ess. ind $\Lambda_{o}>2$, then any $\Lambda_{o}$-Abelian 
integral of the first kind which is reducible with respect to $\left\{A_{j}, B_{j}\right\}_{j \in J}$ is singlevalued on a subregion of infinite genus.

\section{Department of Mathematics KYOTO UNIVERSITY}

\section{References}

[1] L. V. Ahlfors and L. Sario, Riemann surfaces, Princeton Univ. Press, Princeton, 1960, $382 \mathrm{pp}$.

[2] P. Appell et E. Goursat, Théorie des fonctions algébriques et de leurs intégrales, Tome I, Étude des fonctions analytiques sur une surface de Riemann, Gauthier-Villars, Paris, 1929, 526 pp. (Chelsea, New York, 1976.)

[ 3 ] H. F. Baker, Abel's theorem and the allied theory including the theory of theta functions, Cambridge Univ. Press, Cambridge, 1897, 684 pp.

[4] H. Behnke und F. Sommer, Theorie der analytischen Funktionen einer komplexen Veränderlichen, Springer, Berlin-Göttingen-Heidelberg, 1955, 582 pp.

[5] S. Kowalevski, Über die Reduction einer bestimmten Klasse Abel'scher Integrale 3ten Ranges auf elliptische Integrale, Acta math. 4 (1884), 393-414.

[6] A. Krazer, Lehrbuch der Thetafunktionen, Teubner, Leipzig, 1903, 509 pp. (Chelsea, New York, 1970.)

[7] Y. Kusunoki, Theory of functions - Riemann surfaces and conformal mappings, (in Japanese) Asakura, Tokyo, 1973, 408 pp.

[ 8 ] K. Matsui, Convergence theorems of Abelian differentials with applications to conformal mappings, I. J. Math. Kyoto Univ. 15 (1975), 73-100; II. Ibid. 17 (1977), 345-374.

[9] F. Severi, Vorlesungen über algebraische Geometrie. Geometrie auf einer Kurve, Riemannschen Flächen, Abelsche Integrale, (translated by Löfeler, E.) Teubner, Berlin-Leipzig, 1921, 408 pp. (Johnson, New York, 1968).

[10] M. Shiba, Some general properties of behavior spaces of harmonic semiexact differentials on an open Riemann surface. Hiroshima Math. J. 8 (1978), 151-164.

[11] - Abel's theorem for analytic mappings of an open Riemann surface into compact Riemann surfaces of genus one. J. Math. Kyoto Univ. 18 (1978), 305-325.

[12] K. Weierstrass, Letter to L. Königsberger dated October $22^{\text {ndt}}$, 1864, Briefe von K. Weierstrass an L. Königsberger, Acta math. 39 (1923), 226-229.

[13] W. Wirtinger, Algebraische Funktionen und ihre Integrale, Enzyklop. d. math. Wiss. II $B_{2}(1901), 115-175$. 